\title{
Liposarcoma pleomórfico, reporte de caso
}

Pleomorphic liposarcoma, case report

\section{Lipossarcoma pleomórfico, relato de caso}

\author{
Laura Vanessa Ramírez-Pedroza,* \\ Karen Andrea Mantilla-Viviescas, MD.** \\ Federico Guillermo Lubinus-Badillo, MD., Esp. ***
}

\section{Resumen}

Introducción: El liposarcoma pleomórfico es el subtipo con mayor malignidad, presenta recurrencia local de $37 \%$ y metástasis en 10 años de $41 \%$, porcentaje que aumenta directamente con el tamaño de la lesión; además, presenta una supervivencia a los 10 años de $39 \%$. Objetivo: Presentar un caso de esta enfermedad en un hombre sin antecedentes de importancia quien presentó metástasis a pulmón posterior al diagnóstico y tratamiento de un liposarcoma pleomórfico. Discusión: En el grupo de los liposarcomas se encuentran 4 tipos diferentes: El liposarcoma bien diferenciado, desdiferenciado, pleomórfico y mixoide. A pesar de tener poca vascularización el porcentaje de metástasis, su pronóstico y dificultad para extirpación quirúrgica varía según el tipo histológico. En cuanto el diagnóstico: la radiografía simple, la ecografía, la Tomografía computarizada (TC) y la resonancia magnética (RM) son herramientas diagnósticas válidas para el estudio del liposarcoma. El tratamiento es controvertido debido a la tasa de recurrencia después de la resección quirúrgica, la cual oscila entre 0-69\%. Conclusión: En pacientes con cuadros clínicos característicos de lesiones tumorales es importante hacer un diagnóstico oportuno por medio de herramientas imagenológicas y quirúrgicas como el caso de biopsias intraoperatoria para así clasificarlo según el subtipo de liposarcoma, evaluar el tratamiento de elección y evaluar pronóstico y sobrevida del paciente. [RamírezPedroza LV, Mantilla-Viviescas KA, Lubinus-Badillo FG. Liposarcoma pleomórfico. MedUNAB 2017; 19(3): 230-234]

Palabras clave: Liposarcoma; Metástasis de la Neoplasia; Sarcoma; Radiología; Técnicas y Procedimientos Diagnósticos.

\begin{abstract}
Introduction: The pleomorphic liposarcoma is a subtype of liposarcoma with the highest malignancy, with a local recurrence of $37 \%$ and a metastasis in 10 years of $41 \%$, a percentage that increases directly with the size of the lesion; furthermore, it presents a 10-year survival of 39\%. Objective: To show a case of this disease in a man with no relevant background who presented lung metastasis after diagnosis and treatment of the pleomorphic liposarcoma. Discussion: In the group of liposarcomas there are 4 different types: Welldifferentiated, dedifferentiated, pleomorphic and myxoid liposarcoma. In spite of having little vascularization the percentage of metastases, its prognosis and difficulty for surgical removal varies according to its histological type. Regarding to the diagnosis: simple x-ray, ultrasound, computed tomography (CT) and magnetic resonance imaging
\end{abstract}

\footnotetext{
* Estudiante programa de Medicina XI semestre. Universidad Autónoma de Bucaramanga, Floridablanca, Santander, Colombia.

** Médico general, Fundación Oftalmológica de Santander, Floridablanca, Santander, Colombia.

*** Médico y cirujano, especialista en Radiología e Imágenes Diagnósticas. Clínica Carlos Ardila Lülle, Floridablanca, Santander, Colombia
}

Correspondencia: Karen Andrea Mantilla-Viviescas, Universidad Autónoma de Bucaramanga, Calle 157 19-55 Campus El Bosque, Cañaveral, Floridablanca, Santander, Colombia. Correo electrónico: karenmantilla2@hotmail.com. 
(MRI) are valid diagnostic tools to study the liposarcoma. Its treatment is controversial because of the recurrence rate after a surgical resection, which ranges from $0 \%$ to $69 \%$. Conclusion: In patients with clinical profiles characterized by tumor lesions, it is important to make a timely diagnosis using imaging and surgical tools such as intraoperative biopsies to classify the sample according to the liposarcoma subtype, to evaluate the correct treatment, its prognosis and patient survival. [Ramírez-Pedroza LV, Mantilla-Viviescas KA, Lubinus-Badillo FG. Pleomorphic liposarcoma (PLS). MedUNAB 2017; 19(3): 230-234]

Keywords: Liposarcoma; Neoplasm Metastasis; Sarcoma; Radiology; Diagnostic Techniques and Procedures.

\section{Resumo}

Introdução: O lipossarcoma pleomórfico é um subtipo com maior malignidade e apresenta recorrência local de $37 \%$ e metástase em10 anos de $41 \%$, o que aumenta diretamente com o tamanho da lesão. Ele apresenta uma sobrevida de $39 \%$ em 10 anos. Objetivo: Apresentar um caso desta doença num homem sem história de importância que

\section{Introducción}

Se presenta el caso de un paciente con diagnóstico de liposarcoma pleomórfico en miembro inferior, el cual, hizo metástasis años después a su diagnóstico inicial. Este caso clínico es relevante ya que este tipo de liposarcoma, además, de representar solo entre el 10 al 20\% de todos los sarcomas de tejidos blandos y de encontrarse con más frecuencia en extremidades y retroperitoneo (1), representa al tipo de liposarcoma con mayor malignidad con una recurrencia local de $37 \%$ y metástasis en 10 años de $41 \%$, porcentaje que aumenta directamente con el tamaño de la lesión, además de presentar una supervivencia a los 10 años de 39\% (2). En cuanto al diagnóstico la radiografía simple, la ecografía, la Tomografía Computarizada (TC) y la Resonancia Magnética (RM) son herramientas diagnósticas válidas para el estudio del liposarcoma y la naturaleza del tumor se confirma por la hipodensidad característica del tejido adiposo; si se observa tumor blando con contenido lipomatoso en la mayor parte del volumen, durante el diagnóstico imagenológico que se realiza antes de una operación, se piensa en un liposarcoma bien diferenciado con buen pronóstico y si es asintomático no necesita resección quirúrgica. Los tumores de menos de $5 \mathrm{cms}$ y con forma superficial pueden someterse a biopsias con aguja gruesa para hacer diagnóstico histológico (2-3). En cuanto al tratamiento, además de la cirugía, la radioterapia y la quimioterapia son una alternativa en el manejo paliativo de los tumores no operables o en caso de resección incompleta (2).

Es importante desde el inicio tener en cuenta los síntomas y signos que indiquen el comienzo de una lesión tumoral para apresentou metástase no pulmão após o diagnóstico e tratamento do lipossarcoma pleomórfico. Discussão: No grupo de lipossarcoma há 4 tipos diferentes: O lipossarcoma bem diferenciado, indiferenciada, pleomorfo e mixoide. Apesar de ter pouca vascularização a percentagem de metástase, seu prognóstico e dificuldade de remoção cirúrgica varia segundo o tipo histológico. Quanto ao diagnóstico: radiografia simples, ultra-som, tomografia computadorizada (TC) e ressonância magnética (MRI) são ferramentas de diagnóstico válidos para o estudo de lipossarcoma. O tratamento é controvertido porque a taxa de recorrência após a cirúrgia, varia entre 0-69\%. Conclusão: Nos pacientes com diagnosticos clínicos caracterizados pelas lesões tumorais é importante fazer um diagnóstico a tempo, através de instrumentos da imagem e quirúrgicos, como o caso de biópsias intra-operatórias para classificar de acordo com o subtipo de lipossarcoma, avaliar o tratamento a escolhar e avaliar o prognóstico e sobrevivência do paciente. [RamírezPedroza LV, Mantilla-Viviescas KA, Lubinus-Badillo FG. Lipossarcoma pleomórfico. MedUNAB 2017; 19(3): 230-234]

Palavras-chave: Lipossarcoma; Metástase Neoplásica; Sarcoma; Radiologia; Técnicas e Procedimentos Diagnósticos.

así diagnosticarlo de manera temprana, lo que permite brindar un manejo adecuado y oportuno; de esta manera, disminuye la tasa de metástasis y aumenta el porcentaje de supervivencia en los pacientes (1-3).

El objetivo del artículo es presentar un caso típico de esta enfermedad en un hombre sin antecedentes de importancia quien presentó metástasis a pulmón posterior al diagnóstico y tratamiento del liposarcoma pleomórfico.

\section{Presentación del caso}

Paciente masculino de 65 años de edad sin antecedentes personales ni familiares de importancia. Ingresó a urgencias de nuestra institución en enero de 2012 por presentar dolor intenso, incapacitante en cara interna de tercio distal del muslo izquierdo con presencia de masa de crecimiento progresivo; se realizó ecografía de miembro inferior que reportó lesión tumoral expansiva localizada en el interior del vientre muscular del vasto izquierdo. En abril de 2013 se realizó cirugía en la cual se tomó una biopsia que reportó liposarcoma pleomórfico de alto grado de localización Intramuscular profunda: tamaño de $6 \times 4.5 \times 4 \mathrm{~cm}$, conteo mitótico mayor de 20 en 10 campos de alto poder, necrosis del $20 \%$. Histiocitoma fibroso maligno-like sin invasión linfovascular. El paciente fue manejado con quimioterapia coadyuvante y continuó en tratamiento por el servicio de ortopedia oncológica de manera ambulatoria. En noviembre de 2013 asistió al servicio de urgencias por presentar dolor lumbar intenso incapacitante del lado izquierdo 9/10 en escala análoga del dolor, el cual se exacerbaba a la marcha o actividad física asociado a disestesias y dolor tipo urgente en 
extremidad inferior izquierda. Al examen físico se encontró masa de gran tamaño en región lumbar izquierda con disminución de fuerza muscular en miembros inferiores (Fuerza 4/5), se decidió realizar radiografía de pelvis y columna lumbosacra llevó al diagnóstico de discopatía crónica agudizada y se dio salida con antiinflamatorio, analgésico y orden de resonancia magnética de columna lumbosacra de manera ambulatoria.

En abril de 2014 fue remitido desde el servicio de oncología al servicio de urgencias por presentar dolor intenso en región lumbar izquierda con progresión de crecimiento de la masa, con RNM de febrero de 2014 que evidenció lesión expansiva tumoral paravertebral compatible con metástasis, por lo que se decidió realizar nueva RNM con contraste en la cual se confirmó la presencia de una lesión infiltrante de localización paravertebral izquierda con una longitud de $13.0 \mathrm{~cm}$ del nivel de T12 hasta L4, infiltró la musculatura paraespinal, la lámina izquierda, el aparato epifisiario y el pedículo izquierdo de L1 que penetra a través del agujero de conjugación al canal raquídeo en forma parcial (figura 1). Se realizó biopsia trucut que reporta liposarcoma pleomórfico (De alto grado), y se decidió realizar TC de tórax y abdomino-pélvico para descartar metástasis a este nivel (figura 2) en la cual se confirma la metástasis a nivel paravertebral izquierda.

Se decidió hospitalizar en servicio de hematooncología e iniciar quimioterapia neoadyuvante terminando el último

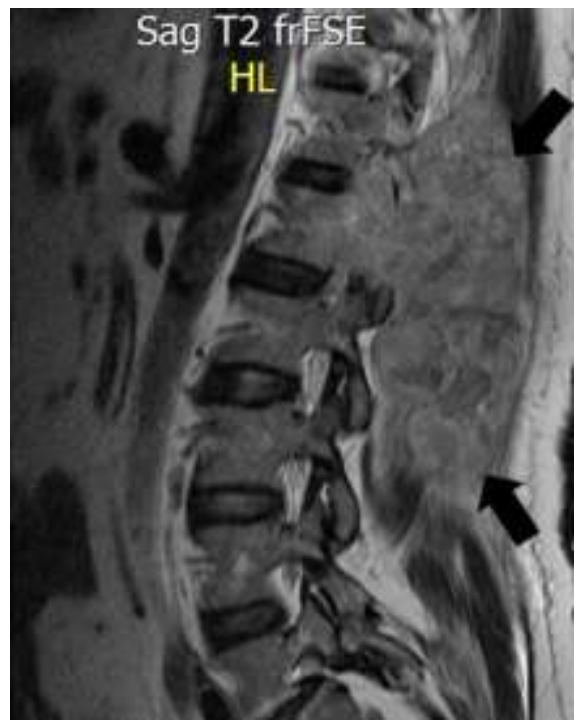

Figura 1. Resonancia nuclear magnética de columna lumbo-sacra, lesión infiltrante de localización paravertebral izquierda que tiene una longitud de $13.0 \mathrm{~cm}$ del nivel de T12 hasta L4, infiltra la musculatura paraespinal, la lámina izquierda, el aparato epifisiario y el pedículo izquierdo de L1 que penetra a través del agujero de conjugación al canal raquídeo en forma parcial. ciclo en mayo, continuando con radioterapia ambulatoria terminando la última sesión en junio de 2014. Continuó en control ambulatorio por el servicio de ortopedia oncológica.

En agosto de 2014 ingresó al servicio de urgencias por fuerte dolor lumbar por lo que se solicitó interconsulta por oncología donde por alta sospecha de recaída reinician ciclos de quimioterapia. Se realizó gammagrafía ósea para descartar metástasis a nivel óseo que reportó hallazgos compatibles con metástasis óseas en ambos fémures y húmero izquierdo, captación en $7^{\circ}$ arco costal derecho sugestivo de metástasis. Desde ese momento, el paciente recibió diagnóstico de liposarcoma pleomórfico metastásico refractario en tratamiento paliativo, Se explicó a familiares quienes decidieron salida voluntaria y continuar manejo paliativo de manera ambulatoria. Paciente murió en enero de 2016 por paro cardiorrespiratorio secundario a falla multiorgánica.

Para la presentación del caso clínico anterior se solicitó y obtuvo consentimiento informado escrito y firmado por los familiares.

\section{Discusión}

Los sarcomas de tejidos blandos son infrecuentes y surgen a partir de los tejidos mesenquimales, pueden ocurrir en cualquier parte del cuerpo y representan el 1\% de todos los

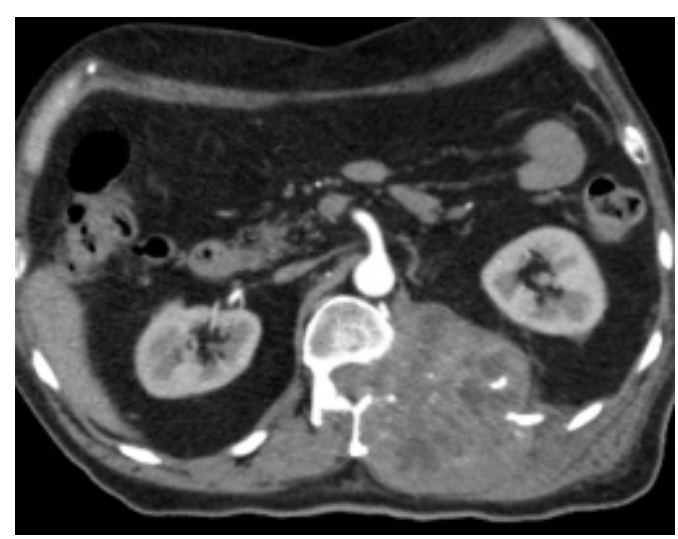

Figura 2. TC Adomino-Pélvico Se observa una lesión expansiva de localización paravertebral izquierda, toraco lumbar que se extiende desde T12 hasta el nivel de L3, muestra una densidad heterogénea, condiciona lisis ósea en el pedículo izquierdo de L1 y se introduce de manera parcial al agujero de conjugación L1-L2. Muestra un patrón de realce anular y heterogéneo moderado. Mide 11.0 x 8.0 x $6.0 \mathrm{~cm}$. Este tumor presenta una imagen cálcica nodular en su aspecto superior y lateral, en aparente relación con pequeño fragmento remanente de la apófisis transversa izquierda de L1. 
tipos de cáncer en adultos (1,2). El 60\% se originan en las extremidades, pero es tres veces más frecuente en miembros inferiores que en los superiores, el 30\% se localizan en el tronco y un $40 \%$ son retroperitoneales(3), estos últimos se presentan en el $10 \%$ de los casos a causa de una metástasis por vía hematógena y el $90 \%$ de novo(2).

El liposarcoma surge a partir de precursores de adipocitos, representa entre el 10-20\% de todos los sarcomas de tejidos blandos y se encuentran con más frecuencia en extremidades y retroperitoneo(4).

Clínicamente, los sarcomas de tejidos blandos representan un grupo muy heterogéneo que tiene en común la aparición de una masa de tejido blando que aumenta de tamaño, mide más de $5 \mathrm{~cm}$ y es dolorosa, entre más características de las mencionadas tenga la lesión, mayor el riesgo de malignidad (5). El tumor puede limitar el rango de movimiento o causa entumecimiento o dolor si se ejerce presión sobre los nervios cercanos(4).

En el grupo de los liposarcomas encontramos 4 tipos diferentes: el liposarcoma bien diferenciado, desdiferenciados, pleomórfico y mixoide. A pesar de tener poca vascularización, el porcentaje de metástasis, su pronóstico y dificultad para extirpación quirúrgica varía según el tipo histológico. En cuanto a factores pronósticos es importante mencionar la relación con los planos aponeuróticos y el tamaño del tumor primario, así como la diferenciación celular, el número de mitosis y la extensión de la necrosis(4).

El liposarcoma bien diferenciado o tumor lipomatoso atípico tiene bajo grado de malignidad que raramente produce metástasis, pero se debe llevar un seguimiento estricto del paciente porque puede producir recurrencia o mala diferenciación (1); así mismo, un liposarcoma mal diferenciado puede aparecer de nuevo o en el $10 \%$ de los casos como complicación a un tumor bien diferenciado preexistente y puede ser de bajo o alto grado(6).

El liposarcoma mal diferenciado se define como una lesión que carece de diferenciación lipogénica con el grado de diferenciación en base a la celularidad, atipia y actividad micótica. El 65\% de los casos ocurren en el retroperitoneo y el $20 \%$ en extremidades $(6)$.

Es un tumor muy agresivo debido a que presenta una tasa de recidiva local de $41 \%$, tasa de metástasis de $17 \%$, tasa de mortalidad 28\%(1) y una tasa de supervivencia a los 5 años de aproximadamente $55 \%$ la cual está definida por la ausencia de la progresión de la enfermedad y no por el grado de respuesta al tratamiento(5).

El liposarcoma mixoide usualmente es una enfermedad indolente, un pequeño porcentaje de pacientes desarrollan metástasis en tejidos blandos años después del diagnóstico inicial lo cual representan un peor pronóstico. Debe ser manejado agresivamente para mejorar la supervivencia ya que presentar recidiva local va a suceder siempre que no se encuentren bien encapsulados o no sean resecados totalmente(3).

En el caso clínico presentado previamente se hizo diagnóstico de liposarcoma pleomórfico, el cual es el subtipo con mayor malignidad, presenta recurrencia local de $37 \%$ y metástasis en 10 años de $41 \%$, porcentaje que aumenta directamente con el tamaño de la lesión, además, presenta una supervivencia a los 10 años de 39\%, en comparación con los demás subtipos que presentan una supervivencia del $87 \%$ cuando son bien diferenciados y $76 \%$ en los liposarcomas mixoides(3).

Las técnicas de imagen juegan un papel muy importante en el diagnóstico de los liposarcomas pleomórficos, entre estas, la tomografía axial computarizada y la resonancia magnética, con estas, podemos diferenciar un liposarcoma bien diferenciado de un liposarcoma pleomórfico según la destrucción ósea y la invasión de tejidos adyacentes(7).

La radiografía simple, la ecografía, la TC y la $\mathrm{RM}$ son herramientas diagnósticas válidas para el estudio del liposarcoma y la naturaleza del tumor se confirma por la hipodensidad característica del tejido adiposo. En la RM, estas lesiones tienen una densidad superior a la de la grasa normal (cercana a la del agua y músculo) y en la TC, la densidad de estos tumores es mezcla de grasa, músculo y agua lo cual puede simular un quiste benigno o un absceso y nos dificultaría el diagnóstico. Cuando usamos la RM, podemos diferenciar tejidos específicos adyacentes y, por tanto, hacer una estadificación tumoral(3).

Las imágenes diagnósticas se realizan antes de una operación, si se observa tumor blando con contenido lipomatoso en la mayor parte del volumen se piensa en un liposarcoma bien diferenciado con buen pronóstico y si es asintomático no necesita resección quirúrgica. Los tumores de menos de $5 \mathrm{cms}$ y con forma superficial pueden someterse a biopsias (3) con aguja gruesa para hacer diagnostico histológico (1). Este procedimiento puede ser realizado de manera preoperatoria o intraoperatoria, siendo esta última la herramienta usada en el paciente presentado en el caso clínico, ya que tiene mayor predilección para la evaluación de liposarcomas y masas de tejido blando, también es útil para hacer una planificación de la resección tumoral y evaluar si hay órganos adyacentes implicados(8).

El tratamiento es controvertido debido a la tasa de recurrencia después de la resección quirúrgica la cual oscila entre $0-69 \%$, Sin embargo, para los casos recurrentes de liposarcoma bien diferenciado es de preferencia hacer una amplia resección para disminuir la probabilidad de mala diferenciación debido a que los márgenes del tumor no son claros(3) y además se debe a que entre más tiempo se deje in situ, mayor probabilidad de diferenciación, en promedio, el tiempo es de 7 a 8 años (1), por ejemplo, en el caso de un liposarcoma retroperitoneal se debe hacer una resección 
completa siempre que sea posible de todas las estructuras adyacentes como el psoas, pared abdominal o músculos paravertebrales(2).

Los principales objetivos terapéuticos en el diagnóstico de liposarcomas son la supervivencia a largo plazo, la prevención de la recurrencia local y disminución de la morbilidad (3). En el caso de lesiones tumorales en extremidades y tronco, el tratamiento de elección es cirugía conservadora combinada con radioterapia pre o post operatoria con lo que se alcanzan altas tasas de control local (5); estos dos elementos tienen resultados locales excelentes y un pequeño riesgo de metástasis en el caso de ser un liposarcoma bien diferenciado o de tipo mixoide, sin embargo, en el caso de ser un liposarcoma pleomórfico, como el caso del paciente presentado, son una herramienta terapéutica válida pero presentan menor expectativa en cuanto a resultados satisfactorios (3) y cuando son a nivel retroperitoneal es más difícil la resección quirúrgica y a veces imposible por las estructuras adyacentes(2).

La radioterapia y la quimioterapia son una alternativa en el manejo paliativo de los tumores no operables o en caso de resección incompleta (3). La recurrencia local es común y constituye la causa de muerte más frecuente en estos casos (2). La radioterapia es adecuada a pesar de la resistencia evidenciada en los tumores mesodérmicos, ya que, de estos, los liposarcomas son los más radiosensibles, por otro lado, la quimioterapia tiene menos resultados positivos en los diferentes subtipos de liposarcomas a excepción del tipo mixoide; sin embargo, es importante considerar el uso de todas las herramientas posibles para mejorar la supervivencia a largo plazo del paciente $(2,3)$.

Debemos tener en cuenta la evolución clínica de la enfermedad, los síntomas y signos clínicos en los pacientes que puedan ser las primeras manifestaciones de una metástasis extra pulmonar o pulmonar para así realizar una detección oportuna(3) y un manejo temprano de la lesión.

Para el seguimiento de los pacientes es necesario realizar resonancias magnéticas o tomografía axial computarizada (5) cada 3-4 meses, durante los primeros 2-3 años, luego, 2 veces al año durante un máximo de 5 años y por último, cada año para completar 8-10 años(5) debido a su alto porcentaje de recidivas(3).

\section{Conclusión}

En pacientes con cuadros clínicos característicos de lesiones tumorales es importante hacer un diagnóstico oportuno por medio de herramientas imagenológicas y quirúrgicas como el caso de biopsias intraoperatorias para así clasificarlo según el subtipo de liposarcoma, evaluar el tratamiento de elección y evaluar pronóstico y sobrevida del paciente.

\section{Conflicto de intereses}

Los autores manifiestan que no tienen ningún conflicto de interés.

\section{Referencias}

1. Nagano S, Yokouchi M, Setoguchi T, Ishidou Y, Sasaki H, Shimada H, Komiya S. Differentiation of lipoma and atypical lipomatous tumor by a scoring system: implication of increased vascularity on pathogenesis of liposarcoma. BMC Musculoskelet Disord 2015;16:36. Doi: 10.1186/ s12891-015-0491-8

2. Matthyssens LE, Creytens D, Ceelen WP. Retroperitoneal liposarcoma: current insights in diagnosis and treatment. Front Surg 2015; 2:4. Doi: 10.3389/fsurg. 2015.00004

3. Calleja-Subirán MC, Hernández-Gutiérrez FJ, LópezElzaurdia C, Revestido-García R. Subtipos histológicos de liposarcoma: presentación de cuatro casos. An Med Interna 2007; 24(4):179-184.

4. Ryan CW, Meyer J. Clinical presentation, histopathology, diagnostic evaluation, and staging of soft tissue sarcoma. In: UpToDate. [Citado 5 feb 2016]. Disponible en: https:// www.uptodate.com/contents/ clinical-presentationhistopathology-diagnostic-evaluation-and-staging-of-softtissue-sarcoma

5. Dangoor A, Seddon B, Gerrand C, Grimer R, Whelan J, Judson I. UK guidelines for the management of soft tissue sarcomas. Clin Sarcoma Res 2016; 6:20. Doi: 10.1186/s13569-016-0060-4

6. Zajicek AK, Bridge JA, Akers JW, McGarry SV, Walker CW. Dedifferentiated liposarcoma of the lower extremity with low-grade dedifferentiation and low-grade osteosarcomatous component. Skeletal Radiol 2017; 46(2):265-271. Doi: 10.1007/s00256-016-2542-0

7. Çizmeci O, Kuvat SV, Karaaltın MV, Bilgiç B. Slowgrowing giant liposarcoma in the extremity: a case report. Eur J Plast Surg 2013; 36(3):207-210. Doi: 10.1007/s00238-011-0674-y

8. Nikolaidis P, Silverman SG, Cibas ES, Vansonnenberg E, Rybicki FJ, Manola JB, Tuncali K, Karshbaum SH, Singer S, Fletcher CD, Demetri GD. Liposarcoma subtypes: identification with computed tomography and ultrasoundguided percutaneous needle biopsy. Eur Radiol 2005; 15(2):383-389. Doi: 10.1007/s00330-004-2501-3 